\title{
Vitiligo and social acceptance
}

This article was published in the following Dove Press journal:

Clinical, Cosmetic and Investigational Dermatology

\section{Reza Bidaki' \\ Niloofar Majidi² \\ Amir Moghadam Ahmadi ${ }^{3}$ \\ Hamid Bakhshi ${ }^{4}$ \\ Rezvan Sadr Mohammadi ${ }^{5}$ \\ Seyed-Ali Mostafavi ${ }^{6}$ \\ Mohammad Kazemi \\ Arababadi ${ }^{7}$ \\ Maryam Hadavi ${ }^{8}$ \\ Afshin Mirzaei ${ }^{9}$}

'Research Center of Addiction and Behavioral Sciences, Diabetes

Research Center, Shahid Sadoughi

University of Medical Sciences, Yazd, Iran; ' 2 Department of Psychiatry,

Rafsanjan University of Medical

Sciences, Rafsanjan, Iran; ${ }^{3}$ Department

of Neurology, Rafsanjan University

of Medical Sciences, Rafsanjan, Iran;

${ }^{4}$ Continuing Medical Education Center,

Rafsanjan University of Medical

Sciences, Rafsanjan, Iran; ${ }^{5}$ Department of Psychiatry, Lorestan University of

Medical Sciences, Khorramabad, Iran;

${ }^{6}$ Psychiatry Research Center, Roozbeh

Hospital, Tehran University of Medical

Sciences, Tehran, Iran; ${ }^{7}$ Department

of Laboratory Sciences, Faculty of

Paramedicine, Rafsanjan University of Medical Sciences, Rafsanjan, Iran; ${ }^{8}$ Department of Anesthesiology, Paramedical Faculty, Molecular Medicine Research Center, Rafsanjan University of Medical Sciences,

Rafsanjan, Iran; ' Rafsanjan University

of Medical Sciences, Rafsanjan, Iran

Correspondence: Maryam Hadavi Department of Anesthesiology, Paramedical Faculty, Molecular Medicine Research Center, Rafsanjan University of Medical Sciences, Rafsanjan, 77/8796755, Iran

Tel +983434258397

Email hadavimaryam@yahoo.com
Background and aim: Vitiligo is a chronic skin disease characterized by a total or partial loss of melanocytes from the epidermis and other tissues of the skin. It is placed in the class of secondary psychiatric disorders and can also lead to psychological problems. The main aim of this study was to assess social acceptance in vitiligo patients.

Methods: This cross-sectional study was conducted on all of the patients $(n=150)$ with vitiligo who were referred to dermatology clinics in Rafsanjan, Iran. The patients completed a social acceptability questionnaire (Marlowe-Crowne Social Desirability Scale), and information regarding their demographic characteristics was also collected. Data were gathered and analyzed with descriptive and inferential statistics using SPSS-19 software.

Results: The mean age of the patients was $27.56 \pm 10.53$ years and $65.9 \%$ were female. Mean score of social acceptance among the patients was $13.51 \pm 7.08$. The results showed that the mean scores of social acceptance were significantly lower in women, in those with single marital status, in those with face and neck lesions, and in those with disease duration less than 5 years. Conclusion: The results showed that certain groups of patients with vitiligo are at greater risk of experiencing lower social acceptance.

Keywords: vitiligo, social acceptance, skin disease, secondary psychiatric disorders

\section{Introduction}

Vitiligo is a common pigmentary skin disorder, characterized by a total or partial loss of melanocytes from the epidermis of skin. The prevalence of vitiligo is $0.1 \%-4 \%$ and is estimated to be about $1 \%$ in white individuals. ${ }^{1}$ The incidence of vitiligo is similar between men and women, and half of the cases occur before the age of 20 years. ${ }^{2}$

As a psycho-dermatology disease, vitiligo is one of the aspects of the new series called "secondary psychiatric disorders". ${ }^{3}$ Vitiligo does not impose serious health problems for the patients, but causes serious beauty problems; meanwhile, most doctors do not assess mood and quality of life in these patients.

The psychological burden of this dermatological problem is relatively high. ${ }^{4}$ Researchers report on the burden of vitiligo that $\sim 75 \%$ of those concerned estimate their appearance as moderately to severely intolerable. ${ }^{5}$ Vitiligo reduces the patients' beauty and has deep effects on their private and social life, and causes social malfunction and decreases their quality of life. ${ }^{6}$ This illness lowers their self-esteem and may cause shame in social interactions. ${ }^{7}$ The patients may experience emotional stress, particularly if vitiligo develops on visible areas of the body like face. Some feel embarrassed, shameful (with decreased self-confidence), depressed, or worried about how others will react. ${ }^{7,8}$ A study showed that more than half of the vitiligo patients 
declared that people stare at them, $20 \%$ said that they are labeled, and $25 \%$ said that their disease causes problems in their relationships with strangers. ${ }^{9}$

Social acceptance of chronic diseases such as vitiligo will be encouraged by social network, including family, friends, colleagues, and neighbors. ${ }^{3}$ Social acceptance is a vital aspect in the life of patients with chronic diseases and may be associated with an improved prognosis. ${ }^{10}$ Furthermore, some studies have mentioned the importance of social acceptance in the quality of life of the patients, ${ }^{11}$ while some patients complain that society does not accept them because of their appearance. ${ }^{12}$ It seems that these patients are not properly supported by their families or society. ${ }^{12}$

Based on the fact that social acceptance in the patients is dependent on social culture, the aim of this study was to determine social acceptance of vitiligo patients in Rafsanjan, a city in Iran.

\section{Methods}

This cross-sectional study was done on the vitiligo patients $(n=150)$ referred to the dermatology clinics in Rafsanjan. Among the 150 patients referred to the dermatology clinic, 126 patients responded to the questionnaire; the response rate was $84 \%$. The inclusion criteria were the patients should be suffering from vitiligo and referred to the dermatology clinic and have the ability to understand the concept of questionnaires. Exclusion criterion was unwillingness to participate in the research.

\section{Data gathering tools}

Marlowe-Crowne Social Desirability Scale (MC-SDS) for assessing social acceptance

MC-SDS questionnaire is one of the most widely used scales for detecting social desirability. MC-SDS consists of 33 items with 2 options for each item, yes (1) or no (0). Total score, which is the sum of all scores, was calculated and ranges from 0 to 33; scores between 0 and 8,9 and 19, and 20 and 33 indicate low, moderate, and high levels of social desirability, respectively.

Marlowe-Crowne Social Desirability scale achieves an acceptable internal consistency coefficient of 0.88 and has reported 0.89 for reliability of retest. ${ }^{13}$

\section{Inventory of demographic characteristics}

This inventory included demographic characteristics such as gender, marital status, education, duration of illness, family history, and location of the lesion.

\section{Statistical analysis}

Descriptive statistics are presented as frequency and percentage or mean \pm standard deviation (SD). The data were analyzed with independent $t$-test and ANOVA. $P$-values less than 0.05 were considered significant. Data analyses were performed using SPSS-19 software.

\section{Ethics}

All participants provided written informed consent. In the case of subjects under the age of 18 years, confirmation of written informed consent from the parents or legal guardians was also obtained. The study was approved by the ethics committee board of Rafsanjan University of Medical Sciences.

\section{Results}

This cross-sectional study was conducted on 126 patients with vitiligo ( 43 men and 83 women). The mean and SD age of the patients was $27.56 \pm 10.53$ years (range 13-60 years), and the average duration of the disease was $7.27 \pm 7.76$ years. In terms of educational status, most patients had diploma $(56.3 \%, \mathrm{~N}=71)$ and primary education $(21.4 \%, \mathrm{~N}=27)$. In terms of family history, the majority of patients had no positive family history (88.1\%) for vitiligo. About 70.6\% (89 patients) had diffuse lesions, $10.3 \%$ (13 patients) had lesions on the extremities, and $7.1 \%$ (9 patients) had lesions on their face. Other demographic characteristics of participants are presented in Table 1.

The mean score of social acceptance in the patients was 13.51 \pm 7.08 . The average scores for social acceptance with regard to different subgroups of gender, marital status, educational level, location of lesion, family history, and time since the occurrence of the disease are displayed in Table 2.

Table I Characteristics of participants in the study of vitiligo and social acceptance

\begin{tabular}{lll}
\hline Characteristics & N & $\%$ \\
\hline Gender & & \\
Men & 43 & 34.1 \\
Women & 83 & 65.9 \\
Marital status & & \\
$\quad$ Married & 56 & 44.4 \\
Single & 60 & 47.6 \\
$\quad$ Divorced & 10 & 8 \\
District & & \\
City & 93 & 74.6 \\
Village & 23 & 19 \\
Countryside & 10 & 6.4 \\
\hline
\end{tabular}


Table 2 Maximum, minimum, mean, and standard deviation of social acceptance score based on demographic variables

\begin{tabular}{|c|c|c|c|c|}
\hline Variables & $\begin{array}{l}\text { Social acceptance } \\
\text { (mean } \pm \text { SD) }\end{array}$ & Minimum & Maximum & $P$-value \\
\hline \multicolumn{5}{|l|}{ Age (years) } \\
\hline $13-22$ & $8.65 \pm 6.70$ & 1 & 33 & $<0.00 I^{* *}$ \\
\hline $23-32$ & $13.86 \pm 5.05$ & 7 & 29 & \\
\hline $33-42$ & $16.91 \pm 6.37$ & 6 & 30 & \\
\hline $43-52$ & $20.25 \pm 4.57$ & 15 & 25 & \\
\hline$>53$ & $24.60 \pm 6.88$ & 18 & 33 & \\
\hline \multicolumn{5}{|l|}{ Gender } \\
\hline Men & $15.27 \pm 7.68$ & 1 & 33 & $0.044^{*}$ \\
\hline Women & $12.60 \pm 6.62$ & 1 & 33 & \\
\hline \multicolumn{5}{|l|}{ Marital status } \\
\hline Single & $9.86 \pm 5.81$ & I & 33 & $0.001 * *$ \\
\hline Married & $|7.58 \pm 6.5|$ & I & 33 & \\
\hline Divorced & $12.60 \pm 4.88$ & 7 & 22 & \\
\hline \multicolumn{5}{|l|}{ Education level } \\
\hline Primary & $12.59 \pm 10.63$ & 1 & 33 & 0.850 \\
\hline Higher & $14.75 \pm 8.39$ & 6 & 30 & \\
\hline Diploma & $13.39 \pm 5.99$ & I & 30 & \\
\hline $\mathrm{BSC}$ & $14.57 \pm 3.77$ & 9 & 23 & \\
\hline MSc or higher & 17 & 17 & 17 & \\
\hline \multicolumn{5}{|l|}{ Location of lesion } \\
\hline Face & $7.66 \pm 4.30$ & I & 13 & $<0.00 I^{* *}$ \\
\hline Neck & $7.85 \pm 5.01$ & I & 13 & \\
\hline Extremities & $18.38 \pm 10.09$ & I & 33 & \\
\hline Trunk & $17.87 \pm 8.80$ & 5 & 33 & \\
\hline Diffuse & $13.44 \pm 6.04$ & 7 & 31 & \\
\hline \multicolumn{5}{|l|}{ Family history } \\
\hline Positive & $15.46 \pm 6.39$ & 7 & 27 & 0.258 \\
\hline Negative & $13.25 \pm 7.16$ & I & 33 & \\
\hline \multicolumn{5}{|c|}{ Duration of disease (years) } \\
\hline$<5$ & $10.32 \pm 5.94$ & I & 33 & $0.001 * *$ \\
\hline $6-10$ & $13.96 \pm 6.12$ & I & 27 & \\
\hline $11-15$ & $18.50 \pm 7.62$ & 8 & 30 & \\
\hline$>15$ & $15.94 \pm 4.67$ & 7 & 25 & \\
\hline
\end{tabular}

Notes: $* P<0.05 ; * P \leq 0.001$.

\section{Discussion}

In this study, we have reported the results of a questionnaire distributed to all vitiligo patients referred to dermatology clinics in Rafsanjan, Iran. Other assessment scales have reported the adverse impact of vitiligo on patient quality of life; however, the MC-SDS has never been used in vitiligo patients. The current report is valuable as additional support that vitiligo negatively impacts patient quality of life. Also, this study focused on the social acceptance in patients with vitiligo. The mean score of social acceptance in vitiligo shows low social acceptability for this disease.

In a study by Karelson et al, the quality of life of patients with vitiligo and psoriasis, as case and healthy control groups, was compared between 2009 and 2011. The average score of the Dermatology Life Quality Index (DLQI) in the vitiligo group was 7.4, which was significantly higher than that of healthy controls (DLQI $=0.6) .{ }^{14}$ The average DLQI score obtained for vitiligo patients in Karelson's study was similar to those obtained for studies performed in Belgium (DLQI = $4.9)$, the UK (DLQI = 4.8), and Indonesia (DLQI = 4.4). ${ }^{15-17}$ In contrast, the DLQI score and social acceptance of vitiligo patients was much better in our study. Similar to our study, the mean DLQI score among the vitiligo patients was higher in Japan (9.5) when compared with studies conducted in other parts of the world. ${ }^{18}$

In a study performed by Ghajarzadeh et al in Tehran between 2009 and 2010, the quality of life in 300 patients (100 patients with alopecia areata, 100 patients with psoriasis, and 100 patients with vitiligo) was assessed, which showed that the average DLQI score in the vitiligo patients was $9.6 \pm 4.8 .{ }^{19}$ Another study conducted in 2008 by Dolatshahi et al reported the DLQI score of vitiligo patients to be 16.8. ${ }^{20}$ 
In Karelson et al's study, the quality of life in patients with vitiligo and psoriasis, as case and healthy control groups, was compared between the years 2009 and 2011. The results showed that vitiligo has a greater impact on feelings and relationships in women. They also showed lower quality of life during the active phase/the first episode of the disease. ${ }^{14}$

In an another study that assessed the quality of life in 119 vitiligo patients and 162 psoriasis patients, women (compared to men) had significantly higher DLQI scores (45.6 vs 13.3), and gender was associated with DLQI score only in psoriasis patients. ${ }^{18}$ However, in the study by Ghajarzadeh et al, women had a higher DLQI score compared with men. ${ }^{19}$ Wang et al showed that patients with vitiligo feel embarrassed and, in the selection of clothing, have serious concerns about covering skin imperfections..$^{20,21}$

\section{Conclusion}

This study indicates that certain groups of patients with vitiligo are at greater risk of experiencing a low acceptance and quality of life. Hence, it can be inferred that these patients require more serious efforts to treat the disease. Accordingly, it seems that the psychological interventions in patients who experience a worse quality of life are very important.

\section{Acknowledgment}

We acknowledge all patients with vitiligo who agreed to participate in this survey.

\section{Disclosure}

The authors report no conflicts of interest in this work.

\section{References}

1. Sahni K, Parsad D, Kanwar AJ, Mehta SD. Autologous noncultured melanocyte transplantation for stable vitiligo: can suspending autologous melanocytes in the patients' own serum improve repigmentation and patient satisfaction? Dermatol Surg. 2011;37(2):176-182.

2. Habif TP, Campbell JL, Chapman MS, Dinulos JGH, Zug KA. Skin Disease, Diagnosis and Treatment. Tehran: Lofty Ideas; 2011.
3. Jin $\mathrm{H}$, Atkinson JH, Yu X, et al. Depression and suicidality in HIV/ AIDS in China. J Affect Disord. 2006;94(1):269-275.

4. Grimes PE. New insights and new therapies in vitiligo. JAMA. 2005;293(6):730-735.

5. Basra M, Fenech R, Gatt R, Salek M, Finlay AY. The Dermatology Life Quality Index 1994-2007: a comprehensive review of validation data and clinical results. Br J Dermatol. 2008;159(5):997-1035.

6. Parsad D, Dogra S, Kanwar AJ. Quality of life in patients with vitiligo. Health Qual Life Outcomes. 2003;1(1):1-3.

7. Ahmed I, Ahmed S, Nasreen S. Frequency and pattern of psychiatric disorders in patients with vitiligo. $J$ Ayub Med Coll Abbottabad. 2007;19(3):19-21.

8. Maleki M, Javidi Z, Kyafr B, Saadtian V, Saremi AK. Prevalence of depression in vitiligo patients. $Q J$ Fundamentals Men Health. 2005; 7(25-26):5-11.

9. Porter JR, Beuf AH, Lerner A, Nordlund J. Psychosocial effect of vitiligo: a comparison of vitiligo patients with "normal" control subjects, with psoriasis patients, and with patients with other pigmentary disorders. J Am Acad Dermatol. 1986;15(2):220-224.

10. Sherr L, Lampe F, Fisher M, et al. Suicidal ideation in UK HIV clinic attenders. AIDS. 2008;22(13):1651-1658.

11. Lee J, Strickland D. Malignant melanoma: social status and outdoor work. Br J Cancer. 1980;41(5):757.

12. AlGhamdi KM, Moussa NA, Mandil A, et al. Public perceptions and attitudes toward vitiligo. J Cutan Med Surg. 2012;16(5):334-340.

13. Johnson TP, Fendrich M, Hubbell A. A validation of the Crowne-Marlowe social desirability scale. Paper presented at: 57 th Annual Meeting of the American Association for Public Opinion Research; 2002.

14. Karelson M, Silm H, Kingo K. Quality of life and emotional state in vitiligo in an Estonian sample: comparison with psoriasis and healthy controls. Acta Derm Venereol. 2013;93(4):446-450.

15. Kent G, al-Abadie M. Factors affecting responses on Dermatology Life Quality Index items among vitiligo sufferers. Clin Exp Dermatol. 1996;21(5):330-333.

16. Mashayekhi V, Javidi Z, Kiafar B, et al. Quality of life in patients with vitiligo: a descriptive study on 83 patients attending a PUVA therapy unit in Imam Reza Hospital, Mashad. Indian J Dermatol Venereol Leprol. 2010;76(5):592.

17. Ongenae K, Van Geel N, De Schepper S, Naeyaert J. Effect of vitiligo on self-reported health-related quality of life. $\mathrm{Br} J$ Dermatol. 2005;152(6):1165-1172.

18. Tanioka M, Yamamoto Y, Kato M, Miyachi Y. Camouflage for patients with vitiligo vulgaris improved their quality of life. J Cosmet Dermatol. 2010;9(1):72-75.

19. Ghajarzadeh M, Ghiasi M, Kheirkhah S. Associations between skin diseases and quality of life: a comparison of psoriasis, vitiligo, and alopecia areata. Acta Med Iran. 2012;50(7):511-515.

20. Dolatshahi M, Ghazi P, Feizy V, Hemami MR. Life quality assessment among patients with vitiligo: comparison of married and single patients in Iran. Indian J Dermatol Venereol Leprol. 2008;74(6):700.

21. Wang KY, Wang KH, Zhang ZP. Health-related quality of life and marital quality of vitiligo patients in China. J Eur Acad Dermatol Venereol. 2011;25(4):429-435.
Clinical, Cosmetic and Investigational Dermatology

Publish your work in this journal

Clinical, Cosmetic and Investigational Dermatology is an international, peer-reviewed, open access, online journal that focuses on the latest clinical and experimental research in all aspects of skin disease and cosmetic interventions. This journal is included on PubMed. The manuscript management system is completely online

\section{Dovepress}

and includes a very quick and fair peer-review system, which is all easy to use. Visit http://www.dovepress.com/testimonials.php to read real quotes from published authors 